\title{
Flow-induced morphological instability of a mushy layer
}

Article

Published Version

FELTHAM, D. L. and GRAE WORSTER, M. (1999) Flowinduced morphological instability of a mushy layer. Journal Of Fluid Mechanics, 391. pp. 337-357. ISSN 0022-1120 doi: https://doi.org/10.1017/S002211209900539X Available at https://centaur.reading.ac.uk/35054/

It is advisable to refer to the publisher's version if you intend to cite from the work. See Guidance on citing.

Published version at: http://dx.doi.org/10.1017/S002211209900539X

To link to this article DOI: http://dx.doi.org/10.1017/S002211209900539X

Publisher: Cambridge University Press

All outputs in CentAUR are protected by Intellectual Property Rights law, including copyright law. Copyright and IPR is retained by the creators or other copyright holders. Terms and conditions for use of this material are defined in the End User Agreement.

\section{www.reading.ac.uk/centaur}

\section{CentAUR}

Central Archive at the University of Reading

Reading's research outputs online 


\title{
Flow-induced morphological instability of a mushy layer
}

\author{
By DANIEL L. FELTHAM AND M. GRAE WORSTER ${ }^{1}$ \\ ${ }^{1}$ Department of Chemical Engineering, University of Manchester Institute of Science \\ and Technology, PO Box 88, Manchester M60 1QD, UK \\ ${ }^{2}$ Institute of Theoretical Geophysics, Department of Applied Mathematics \\ and Theoretical Physics, University of Cambridge, Silver Street, Cambridge CB3 9EW, UK
}

(Received 20 March 1998 and in revised form 9 March 1999)

A morphological instability of a mushy layer due to a forced flow in the melt is analysed. The instability is caused by flow induced in the mushy layer by Bernoulli suction at the crests of a sinusoidally perturbed mush-melt interface. The flow in the mushy layer advects heat away from crests which promotes solidification. Two linear stability analyses are presented: the fundamental mechanism for instability is elucidated by considering the case of uniform flow of an inviscid melt; a more complete analysis is then presented for the case of a parallel shear flow of a viscous melt. The novel instability mechanism we analyse here is contrasted with that investigated by Gilpin et al. (1980) and is found to be more potent for the case of newly forming sea ice.

\section{Introduction}

The change of phase from liquid to solid can be significantly influenced by fluid motions of the liquid. For example, there are many circumstances in which the solidliquid interface becomes rippled in the presence of a flow. This has been observed on the underside of ice forming on top of a flowing river (Ashton \& Kennedy 1972), during the freezing of a water pipe (Özisik \& Mulligan 1969) and in a cylindrical annulus with a heated inner wall and a cooled outer wall on which solid has formed (Fang et al. 1985). In the last case, buoyancy forces are primarily responsible for the ripple-forming instability. In the other cases, the instability is due entirely to the externally forced flow. Gilpin, Hirata \& Cheng (1980) have shown that such an instability of a solid-liquid interface requires a phase shift in the range $\pi / 2$ to $\pi$ between the incipient ripples and the heat transfer from the melt. A phase shift in this range can occur in turbulent flow over a wavy surface (Ashton 1972) for certain values of the Reynolds number. Gilpin et al. (1980) used the semi-empirical relationship for heat transfer determined by Ashton (1972) in a linear stability analysis of a planar, stationary, solid-liquid interface and determined conditions on the relative heat transfer from the melt and through the solid for instability to occur. Although this was a linear analysis, it relied on a heat-transfer correlation for flow over a finite-amplitude surface corrugation. Experiments (Gilpin et al.1980) show that quite a large initial perturbation to a planar interface is required in practice to trigger an instability by this mechanism.

In this paper, we analyse the stability of an interface between a mushy layer growing from a flowing melt. A mushy layer is a porous medium comprising a matrix of solid 
crystals through which the residual melt can flow. Mushy layers form very commonly when solid grows from a binary melt or mixture (see Huppert 1990 for examples). Sea ice, which grows from the salty ocean, is a mushy layer with a liquid volume fraction of about $10 \%$. Ice growing from a river containing pollutants or high concentrations of dissolved minerals is also likely to be mushy. We find that an entirely different and truly linear mechanism can cause ripples on the interface between a mushy layer and a melt. The instability we analyse relies on a flow through the mushy layer induced by the Bernoulli effect causing low pressure at the crests of incipient corrugations. The flow interacts with the thermal gradients driving solidification to cause an instability.

In $\S 2$, we write down the model equations we use to describe the mushy layer and fluid region. These have a simple steady solution in which the mush-liquid interface is planar, the mushy layer is stagnant and there is a parallel flow of the liquid. We present perturbation equations and solve them in $\S 3$ for the case of a uniform flow of an inviscid melt in which the no-slip condition is relaxed; this illuminates the fundamental mechanisms controlling the instability. After making a simplification of the model of the mushy region in $\S 4$, which we show is not detrimental to the study of the instability, we discuss the stability of the mush-liquid interface in the presence of a viscous shear flow in $\S 5$. In this analysis, the hydrodynamic modes in the melt (Drazin \& Reid 1981) are suppressed by considering the steady flow past a wavy boundary. This was done in order to focus on the morphological instability induced by the flow which does not require the existence of the hydrodynamic modes and can be triggered at Reynolds numbers lower than that required for their existence. Though we believe the physical mechanisms that we describe are important at higher Reynolds numbers, the existence of the hydrodynamic modes may then render our calculations inaccurate. In $\S 6$, we compare the instability mechanism described in this paper with that of Gilpin et al. (1980), applying both analyses to the case of shear flow beneath growing sea ice. General conclusions of the study are presented in $\S 7$.

\section{Formulation}

The physical situation we consider is shown schematically in figure 1 . We consider a two-dimensional system in which a binary alloy is solidifying at a constant rate $V$. There is a mushy layer in the region $0<z<\zeta(x, t)$ sandwiched between a completely solid region and a completely liquid region. The liquid region is taken to be semi-infinite with a far-field composition $C_{0}$ and temperature $T_{\infty}$ greater than the liquidus temperature $T_{L}\left(C_{0}\right)$, which is the equilibrium freezing temperature of the alloy. We fix our coordinates in a frame of reference moving downwards with the solidification speed $V$ relative to the solid phase. For clarity of presentation, we consider the thermal properties and densities of the solid and liquid phases to be the same. We neglect buoyancy forces, which enables us to focus attention on the effects of forced convection alone. We ignore the diffusion of solute in the mush and liquid, an approximation that is valid if the Lewis number $(L e=\kappa / D)$, the ratio of thermal to solutal diffusivities, is very much greater than unity, which is typical of liquids and solids. The mushy layer is assumed to be in local thermodynamic equilibrium so that the temperature $T$ is related to the composition $C$ via a liquidus relationship, which we assume to be linear; $T=T_{L}(C) \equiv \Gamma C$ where $\Gamma$ is a constant. The equations describing the mushy region are given in detail in Worster (1992a). Here we non-dimensionalize the governing equations by scaling lengths with $\kappa / V$ and 


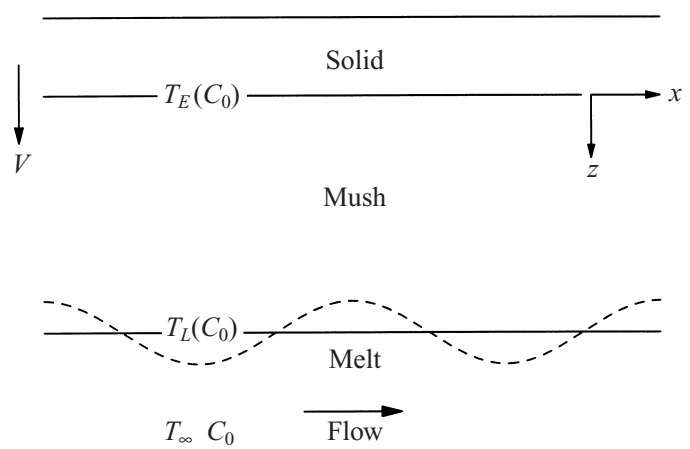

FIGURE 1. The geometry of the mushy-layer system. The mushy layer is sandwiched between a layer of impermeable solid and the melt. The solid-mush interface is assumed planar and is at the eutectic temperature $T_{E}$. The mush-melt interface is at the liquidus temperature $T_{L}\left(C_{0}\right)>T_{E}$. The melt region is semi-infinite with far-field temperature $T_{\infty}>T_{L}\left(C_{0}\right)$ and is of uniform composition $C_{0}$. We consider the mushy layer to advance into the melt at the constant rate $V$. In the basic state, the mush-melt interface is flat and the mushy layer is stagnant; any parallel flow in the melt does not affect this basic state. In the stability analyses, we perturb the mush-melt interface (dashed line) and calculate its linear evolution.

times with $\kappa / V^{2}$. The non-dimensional form of the conservation equations for heat and solute in the mushy layer is then

$$
\begin{gathered}
\left(\frac{\partial}{\partial t}-\frac{\partial}{\partial z}\right)(\theta-\mathscr{S} \phi)+\boldsymbol{u} \cdot \nabla \theta=\nabla^{2} \theta, \\
\left(\frac{\partial}{\partial t}-\frac{\partial}{\partial z}\right)[(1-\phi) \theta+\mathscr{C} \phi]+\boldsymbol{u} \cdot \nabla \theta=0 .
\end{gathered}
$$

In these equations, $\phi$ is the local solid fraction in the mushy layer and $\theta$ represents both the dimensionless temperature and composition:

$$
\theta=\frac{T-T_{L}\left(C_{0}\right)}{\Delta T}=\frac{C-C_{0}}{\Delta C},
$$

where $\Delta T=T_{L}\left(C_{0}\right)-T_{E}$ and $\Delta C=C_{0}-C_{E}$. The dimensionless parameters are the Stefan number

and a composition ratio

$$
\mathscr{S}=\frac{\mathscr{L}}{c \Delta T}
$$

$$
\mathscr{C}=\frac{C_{s}-C_{0}}{C_{0}-C_{E}},
$$

where $\mathscr{L}$ is the latent heat, $c$ is the specific heat capacity, $C_{s}$ is the composition of the solid phase (often nearly zero), and $T_{E}$ and $C_{E}$ are the eutectic temperature and composition respectively.

We take the composition in the liquid region to be uniform and equal to $C_{0}$, while the usual advection-diffusion equation,

$$
\left(\frac{\partial}{\partial t}-\frac{\partial}{\partial z}\right) \theta+\boldsymbol{u} \cdot \nabla \theta=\nabla^{2} \theta,
$$

describes local conservation of heat.

These equations describe the heat and solute fields in the mushy and liquid regions but we also need a specification for the flow in the two regions. In common with 
many previous studies, for example Hills, Loper \& Roberts (1983), Fowler (1985) and Worster (1991), we treat the mushy layer as a porous medium and model the flow through it using Darcy's Law,

$$
\boldsymbol{u}=-\frac{\boldsymbol{\Pi}}{\mu} \nabla p,
$$

where $\mu$ is the dynamic viscosity and $p$ is the pressure. Though the permeability $\Pi$ is in general a second-rank tensor, we consider it here to be locally isotropic, though a function of the local solid fraction, and shall therefore represent it by the scalar $\Pi(\phi)$. The flow in the liquid region is incompressible and governed by the Navier-Stokes equations,

$$
\left(\frac{\partial}{\partial t}-\frac{\partial}{\partial z}\right) \boldsymbol{u}+\boldsymbol{u} \cdot \nabla \boldsymbol{u}=-\frac{1}{\rho} \nabla p+v \nabla^{2} \boldsymbol{u}
$$

where $v=\mu / \rho$ is the kinematic viscosity.

We apply the following boundary conditions to the mush-melt system:

$$
\begin{aligned}
\theta=-1 & (z=0), \\
\theta=0, \quad \phi=0, \quad[\theta]_{l}^{m}=0, & \phi \mathscr{S} v_{n}=[\boldsymbol{n} \cdot \nabla \theta]_{l}^{m} \quad(z=\zeta), \\
\theta \longrightarrow \theta_{\infty} & (z \longrightarrow \infty),
\end{aligned}
$$

where $\theta_{\infty}=\left(T_{\infty}-T_{L}\left(C_{0}\right)\right) / \Delta T$ is the dimensionless far-field temperature, $\boldsymbol{n}$ is the unit normal pointing into the melt, $v_{n}$ is the normal velocity of the interface, $m$ and $l$ refer to quantities belonging to the mushy layer and liquid respectively and []$_{l}^{m}$ refers to the jump across the mush-liquid interface. The condition on the solid fraction $(2.8 c)$ is discussed in Worster (1992b) and follows from an assumption of marginal equilibrium (Worster 1986). Since the solid fraction is zero at the mush-liquid interface, there is no latent heat release at the interface, and the Stefan condition, $(2.8 e)$, simply expresses continuity of heat flux. In $\$ \S 4$ and 5 an approximate model is examined in which the solid fraction is assumed to be uniform in the mushy layer. In this case, the left-hand side of $(2.8 e)$ is retained.

The solid-mush interface is assumed planar and, since it is impermeable, the normal flow there is zero. At the mush-liquid interface, we require continuity of flux and apply continuity of pressure and the no-slip condition on the liquid side. These conditions are

$$
\begin{gathered}
w=0 \quad(z=0), \\
{[\boldsymbol{u} \cdot \boldsymbol{n}]_{l}^{m}=0, \quad[p]_{l}^{m}=0, \quad \boldsymbol{u}^{l} \cdot \tau=0 \quad(z=\zeta),}
\end{gathered}
$$

where $\tau$ is any vector tangent to the interface. We note that replacement of the noslip condition with the Beavers-Joseph boundary condition (Beavers \& Joseph 1967) allows a small slip flow at the mush-melt interface. However, a scaling analysis shows that the slip velocity is insignificant relative to the far-field flow, and we shall ignore it. In $\S 3$, the fundamental mechanism of the instability is explored by considering a uniform flow of an inviscid melt and thus the no-slip condition is abandoned. A viscous melt is considered in the subsequent analysis, however, and the condition is reinstated.

This system admits a steady basic state in which the mush-liquid interface is planar, see figure 1 . We allow for an arbitrary parallel shear flow in the liquid region of the form $\boldsymbol{u}=(u(z), 0)$ that satisfies the no-slip condition but the mushy layer is 
stagnant. Note that the only coupling between the flows in the liquid and mushy layer is through the continuity of normal mass flux and the pressure field. There is therefore no tendency for the parallel flow in the liquid to induce a flow in the mushy layer while the interface remains planar. The solution for the basic state is given in Worster (1992a), the temperature field being given implicitly by an expression of the form $z=f(\theta)$. In order to simplify the stability analysis we assume here that $\mathscr{C} \gg 1$, which allows a linear decoupling of the thermal and solutal field equations in the mush and admits an explicit solution in the mushy layer. This condition is slightly less restrictive than the near-eutectic approximation used by Fowler (1985), Emms \& Fowler (1994) and Anderson \& Worster (1996), which involves the further limit $\theta_{\infty} \gg 1$. The basic-state solution is then

$$
\begin{gathered}
\theta=\frac{\theta_{\infty}}{\Omega}\left(1-\mathrm{e}^{-\Omega(z-\zeta)}\right), \\
\phi=-\frac{\theta}{\mathscr{C}}
\end{gathered}
$$

in the mush and

$$
\theta=\theta_{\infty}\left(1-\mathrm{e}^{-(z-\zeta)}\right)
$$

in the liquid. The mushy-layer depth is

$$
\zeta=\frac{1}{\Omega} \ln \left(1+\frac{\Omega}{\theta_{\infty}}\right)
$$

in which the parameter

$$
\Omega=1+\frac{\mathscr{S}}{\mathscr{C}}
$$

is a measure of the linear coupling of the heat and solute equations in the mushy layer.

A particular, simplifying feature of the limit $\mathscr{C} \gg 1$ is that the solid fraction is small (see equation $(2.10 b)$ ) and the permeability is approximately uniform (Anderson \& Worster 1996). Darcy's equation (2.6) then shows that the velocity field is irrotational in this limit, which is exploited below.

\section{Linear stability analysis with uniform flow in the melt}

The mechanism for instability is most simply revealed by the following analysis in which we assume the liquid external to the mushy layer to be inviscid and its flow to be irrotational. Since the liquid is inviscid, we abandon the no-slip condition. The basic state we perturb is one in which the flow in the liquid region is uniform and parallel to the interface; it is thus not liable to shear instability. Since the instability we investigate is an interfacial instability of the mush-melt interface, it is little affected by the solid-mush interface (as demonstrated by calculations for an infinite mushy layer, Feltham 1997). Thus, for simplicity, perturbations to the solid-mush interface are ignored. We introduce normal modes by writing the dependent variables in the form

$$
(\zeta, \theta, \phi, \boldsymbol{u})=\left(\zeta_{0}, \theta_{0}, \phi_{0}, \boldsymbol{u}_{0}\right)+\epsilon\left(\zeta_{1}, \theta_{1}, \phi_{1}, \boldsymbol{u}_{1}\right) \mathrm{e}^{\sigma t+\mathrm{i} k x}
$$

in which the variables $\theta_{0}$, $\phi_{1}$, etc. are all functions of $z$ alone, $\zeta_{0}$ and $\zeta_{1}$ are constants and the subscript 0 refers to the basic-state solution. Since the flow is taken to be 
irrotational, there is a velocity potential

$$
\Phi^{l}=\mathscr{U} x+\epsilon \Phi_{1}^{l} \mathrm{e}^{\sigma t+\mathrm{i} k x}
$$

in the liquid region and a potential

$$
\Phi^{m}=\epsilon \Phi_{1}^{m} \mathrm{e}^{\sigma t+\mathrm{i} k x}
$$

in the mushy layer, where $\mathscr{U}=U / V$ is the ratio of the forcing flow to the growth rate. These each satisfy Laplace's equation

$$
\left(\mathrm{D}^{2}-k^{2}\right) \Phi_{1}^{m, l}=0,
$$

which is solved in place of (2.6) and (2.7), where $\mathrm{D} \equiv \mathrm{d} / \mathrm{d} z$.

The no-slip condition (2.9d) cannot be applied to the flow of inviscid fluid. The remaining conditions on the flow $(2.9 a-c)$ become

$$
\begin{gathered}
\mathrm{D} \Phi_{1}^{m}=0 \quad(z=0), \\
\mathrm{D} \Phi_{1}^{l}-\mathrm{D} \Phi_{1}^{m}-\mathrm{i} k \mathscr{U} \zeta_{1}=0 \quad\left(z=\zeta_{0}\right), \\
-\frac{P r}{D a} \Phi_{1}^{m}+\mathrm{i} k \mathscr{U} \Phi_{1}^{l}+(\sigma-\mathrm{D}) \Phi_{1}^{l}=0 \quad\left(z=\zeta_{0}\right) .
\end{gathered}
$$

In deriving (3.5c) from $(2.9 c)$, the pressure in the liquid region is determined from linearizing Bernoulli's equation, which is

$$
\rho \frac{\partial \Phi^{l}}{\partial t}+p+\frac{1}{2} \rho\left|\nabla \Phi^{l}\right|^{2}=F(t),
$$

where $F(t)$ is an unknown function of $t$ only. Additionally, we require

$$
\Phi_{1}^{l} \longrightarrow 0 \quad(z \longrightarrow \infty)
$$

The Darcy number

$$
D a=\frac{\Pi}{(\kappa / V)^{2}}
$$

is the ratio of the permeability of the mushy layer (proportional to the square of the interstitial lengthscale) to the square of the diffusion length $\kappa / V$; and the Prandtl number is $\operatorname{Pr}=v / \kappa$. We make use of the fact that $\mathscr{U}$ is typically very large $\left(\approx 10^{4}\right.$ to $\left.10^{5}\right)$ and the Darcy number typically very small $\left(\approx 10^{-8}\right)$ to simplify the boundary conditions $(3.5 b, c)$ to

$$
\mathrm{D} \Phi_{1}^{l} \sim \mathrm{i} k \mathscr{U} \zeta_{1}, \quad \Phi_{1}^{m} \sim \mathrm{i} k \mathscr{U}(D a / P r) \Phi_{1}^{l} \quad\left(z=\zeta_{0}\right) .
$$

The first of these implies that $\Phi_{1}^{l}=O(\mathscr{U})$. With this, the second condition shows that $\Phi_{1}^{m}=O\left(D a \mathscr{U}^{2}\right)$. We proceed under the assumption that $D a \mathscr{U}^{2}=O(1)$ so that, for example, $\Phi_{1}^{m} \ll \Phi_{1}^{l}$ and the asymptotic approximations that led to (3.9) are self-consistent. With these assumptions, the solutions for the velocity potentials are

$$
\begin{gathered}
\Phi_{1}^{l}=-\mathrm{i} \mathscr{U} \zeta_{1} \mathrm{e}^{-k\left(z-\zeta_{0}\right)}, \\
\Phi_{1}^{m}=\frac{D a \mathscr{U}^{2}}{P r} k \zeta_{1} \cosh (k z) / \cosh \left(k \zeta_{0}\right) .
\end{gathered}
$$

We see that $(3.10 a)$ is just the velocity potential for flow over a solid wavy boundary. This is because, on the scale of the flow in the liquid region, the mushy layer is effectively impermeable, as reflected by equation (3.9a). However, this flow has an 
(a)

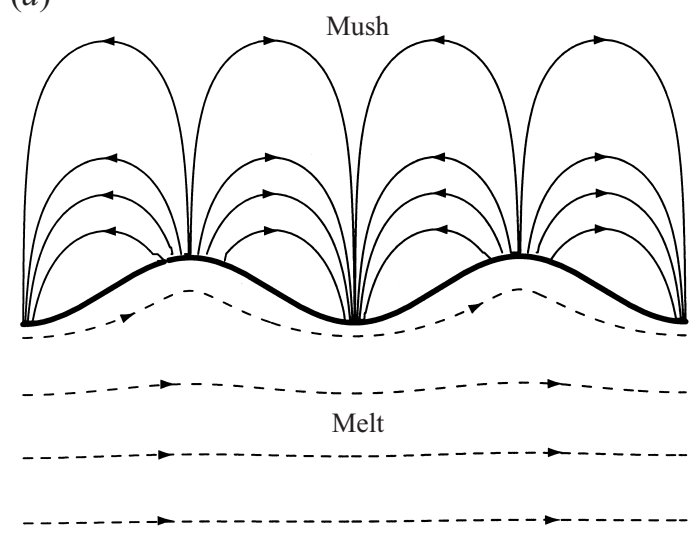

(b)

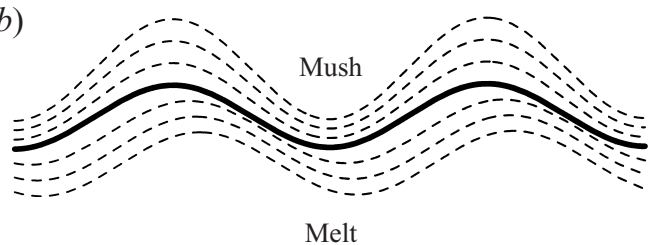

FIGURE 2. (a) Calculated streamlines in the mushy layer and melt. The flow in the mushy layer is negligible compared to the flow in the melt. The contour levels of the streamfunction in the mush are $\pm 2 \times 10^{-2}, \pm 10^{-2}, \pm 5 \times 10^{-3}$ and $\pm 5 \times 10^{-4}$ (decreasing in magnitude away from the interface, positive values to the left of the crests). The contour levels of the streamfunction in the melt are $10^{3}, 3 \times 10^{3}, 5 \times 10^{3}, 7 \times 10^{3}$ and $9 \times 10^{3}$ (increasing in magnitude away from the interface). The flow in the melt is calculated by treating the mush as impermeable and corresponds to flow past a solid wavy boundary. (b) Schematic diagram indicating the effect of the perturbed flow in the mushy layer and melt on the isotherms.

associated pressure field that can drive the weak flow (3.10b) in the mushy layer. It is this latter flow that interacts with the temperature field to produce instability. This is illustrated in figure 2, which shows the streamlines for sample parameter values and a schematic diagram of the effect on the isotherms. The streamlines were calculated for $\mathscr{U}=10^{4}, D a=10^{-8}, \operatorname{Pr}=10, \zeta_{0}=\zeta_{1}=k=1$ and $\epsilon=0.1$. The contour levels in the mush are chosen to reveal the weak flow there; this flow is negligible compared to the flow in the melt. In the liquid region, the isotherms are compressed at the upstream face of each crest and rarefied at the downstream face. This causes the upstream face to melt and the downstream face to freeze, which gives a translation of the interface shape in the direction of the basic flow. Thus, the effect of the flow in the liquid on the amplitude of the interfacial perturbation is neutral. Moreover, the effect of heat conduction is stabilizing so that, overall, laminar flow over a solid-liquid phase boundary does not cause instability. However, the isotherms in the mushy region are compressed near the crests, which enhances conduction of heat away from the mush-liquid interface and promotes its advance. Conversely, the isotherms at the troughs are rarefied and the advance of the interface is retarded. It is this mechanism by which flow over a mush-liquid interface may cause a morphological instability of the interface.

The interactions just described are now quantified by solving for the perturbations 
to the temperature field and interface shape given the flows described by equations $(3.10 a, b)$. By using the approximation that $\mathscr{C} \gg 1$, the perturbed conservation equations for heat and solute in the mushy layer

$$
\begin{gathered}
\left(\mathrm{D}^{2}+\Omega \mathrm{D}-k^{2}-\Omega \sigma\right) \theta_{1}=\Omega w_{1} \mathrm{D} \theta_{0}, \\
(\mathrm{D}-\sigma) \phi_{1}=\frac{1}{\mathscr{C}} w_{1} \mathrm{D} \theta_{0}
\end{gathered}
$$

are uncoupled, while the perturbation equation for conservation of heat in the liquid region is

$$
\left(\mathrm{D}^{2}+\mathrm{D}-k^{2}-\sigma-\mathrm{i} k u_{0}\right) \theta_{1}=w_{1} \mathrm{D} \theta_{0} .
$$

The boundary and interfacial conditions for these equations are

$$
\begin{array}{rlrl}
\theta_{1} & =0 & (z=0), & \\
\theta_{1}+\zeta_{1} \mathrm{D} \theta_{0}=0, & \phi_{1}+\zeta_{1} \mathrm{D} \phi_{0}=0, & {\left[\theta_{1}\right]_{l}^{m}=0,} & {\left[\mathrm{D} \theta_{1}\right]_{l}^{m}+\zeta_{1}\left[\mathrm{D}^{2} \theta_{0}\right]_{l}^{m}=0} \\
\theta_{1} \rightarrow 0 & (z \rightarrow \infty), & \left(z=\zeta_{0}\right),
\end{array}
$$

which are derived from $(2.8 a-f)$.

The eigenfunctions can be found analytically. The temperature in the mushy layer is

$$
\begin{aligned}
\theta_{1}^{m}=\zeta_{1} \theta_{\infty} A\left\{\mathrm{e}^{-\Omega\left(z-\zeta_{0}\right)}\left(\cosh (k z)-\frac{\sigma}{k} \sinh (k z)\right)-\mathrm{e}^{\left(m_{1}+\Omega\right) \zeta_{0}}\right\} & \\
& +\zeta_{1} \theta_{\infty} B\left\{\mathrm{e}^{m_{2}\left(z-\zeta_{0}\right)}-\mathrm{e}^{X \zeta_{0}}\right\},
\end{aligned}
$$

where

$$
\begin{gathered}
A=\frac{-k}{\sigma^{2}-k^{2}} \frac{(D a / P r) \mathscr{U}^{2} k^{2}}{\cosh \left(k \zeta_{0}\right)}, \\
B=\frac{1}{\mathrm{e}^{X \zeta_{0}}-1}\left\{1+A\left(\cosh \left(k \zeta_{0}\right)-\frac{\sigma}{k} \sinh \left(k \zeta_{0}\right)-\mathrm{e}^{\left(m_{1}+\Omega\right) \zeta_{0}}\right)\right\} \\
m_{1,2}=-\Omega / 2 \pm X, \\
X=\frac{1}{2}\left(\Omega^{2}+4 k^{2}+4 \Omega \sigma\right)^{1 / 2} .
\end{gathered}
$$

The temperature in the liquid region is

$$
\theta_{1}^{l}=\zeta_{1} \theta_{\infty} C \mathrm{e}^{-(k+1)\left(z-\zeta_{0}\right)}-\zeta_{1} \theta_{\infty}(1+C) \mathrm{e}^{q\left(z-\zeta_{0}\right)},
$$

where

$$
\begin{gathered}
C=\frac{\mathrm{i} k \mathscr{U}}{(k-\sigma-\mathrm{i} k \mathscr{U})}, \\
q=-\frac{1}{2}-\frac{1}{2}\left(1+4 k^{2}+4 \sigma+4 \mathrm{i} k \mathscr{U}\right)^{1 / 2} .
\end{gathered}
$$

In these expressions for the temperature, the growth rate $\sigma$ (the eigenvalue) is determined from the Stefan condition (3.13e) which, upon substitution of the temperature fields, becomes

$$
(k+1+q) C+q+\alpha+\beta \Upsilon=\mathscr{S} / \mathscr{C},
$$


where

$$
\begin{gathered}
\alpha=\frac{(D a / P r) \mathscr{U}^{2}}{\left(\sigma^{2}-k^{2}\right)}\left\{\mathrm{e}^{\zeta_{0}(\Omega / 2-X)}(\Omega / 2+X) k^{3} / \cosh \left(k \zeta_{0}\right)\right. \\
\left.+k^{2}\left(k^{2}+\Omega \sigma\right) \tanh \left(k \zeta_{0}\right)-k^{3}(\Omega+\sigma)\right\}, \\
\beta=\frac{\left(X \operatorname{coth}\left(\zeta_{0} X\right)-\Omega / 2\right)}{\left(\sigma^{2}-k^{2}\right)}, \\
\Upsilon=k^{2}-\sigma^{2}+(D a / \operatorname{Pr}) \mathscr{U}^{2}\left\{\frac{\mathrm{e}^{\zeta_{0}(\Omega / 2-X)} k^{3}}{\cosh \left(k \zeta_{0}\right)}-k^{2}\left(k-\sigma \tanh \left(k \zeta_{0}\right)\right)\right\} .
\end{gathered}
$$

We note that the growth rate is in general complex: $\sigma=\sigma_{R}+\mathrm{i} \sigma_{I}$, where $\sigma_{R}$ and $\sigma_{I}$ are real. The neutral curve is given by solving $(3.16 a)$ given $\sigma_{R} \equiv 0$ for the unknowns $\mathscr{U}$ and $\sigma_{I}$. This requires the solution of a complex transcendental equation which was performed numerically using a nested Newton-Raphson root-finding algorithm.

Since we expect the perturbed heat flux from the melt into the mushy layer to have a neutral effect on the stability (principally producing a simple translation of the interface shape), we arbitrarily simplify the analysis by ignoring this heat flux and considering the resulting, simplified Stefan condition

$$
\alpha+\beta \Upsilon=\Omega .
$$

Ignoring the perturbed heat flux from the melt removes translation of the interface and gives a direct instability $\left(\sigma_{I} \equiv 0\right)$. In this case, the neutral curve can be found analytically to be

$$
\begin{aligned}
\mathscr{U}_{n}^{2}=\frac{P r}{2 D a k}\left(\Omega+Y \operatorname{coth}\left(\zeta_{0} Y / 2\right)\right) & \left\{-k \tanh \left(\zeta_{0} k\right)+(1 / 2)\left[\Omega+Y \operatorname{coth}\left(\zeta_{0} Y / 2\right)\right.\right. \\
& \left.\left.-Y \mathrm{e}^{\zeta_{0}(\Omega-Y) / 2} \frac{\left(1+\operatorname{coth}\left(\zeta_{0} Y / 2\right)\right)}{\cosh \left(\zeta_{0} k\right)}\right]\right\}^{-1}
\end{aligned}
$$

where

$$
Y=\left(\Omega^{2}+4 k^{2}\right)^{1 / 2}
$$

Note that $\mathscr{U}_{n} \propto(\operatorname{Pr} / D a)^{1 / 2}$.

In figure 3, we show two neutral curves, $(D a / P r)^{1 / 2} \mathscr{U}$ versus $k$. Curve $(a)$ is the solution to the full Stefan condition (3.16a), which includes the perturbed heat flux from the melt and is obtained numerically. Curve $(b)$ ignores the heat flux from the melt, is the solution of (3.17), and is given by (3.18a). We note that curve $(b)$ is independent of $D a$ and $P r$. The parameters used are $D a=10^{-8}, P r=10, \Omega=11$ and $\theta_{\infty}=0.1$.

It is clear that for these parameter values, the effect of the perturbed heat flux from the melt is slight and stabilizing. At large $\mathscr{U}$, the isotherms in the melt are sufficiently deformed that the effect of the heat flux on the mush-melt interface is almost completely to cause translation and is therefore neutral. This is indicated by the curves $(a)$ and $(b)$ coinciding at small $k$. By ignoring the perturbed heat flux from the melt, we obtain a good estimate for the neutral curve which gives a lower bound on the flow rate required for instability to ensue.

The critical flow rate $\mathscr{U}_{\text {crit }}$ is the minimum flow rate required to induce instability which, for potential flow, is also the value of $\mathscr{U}_{n}$ as $k \rightarrow \infty$. If we ignore the perturbed 


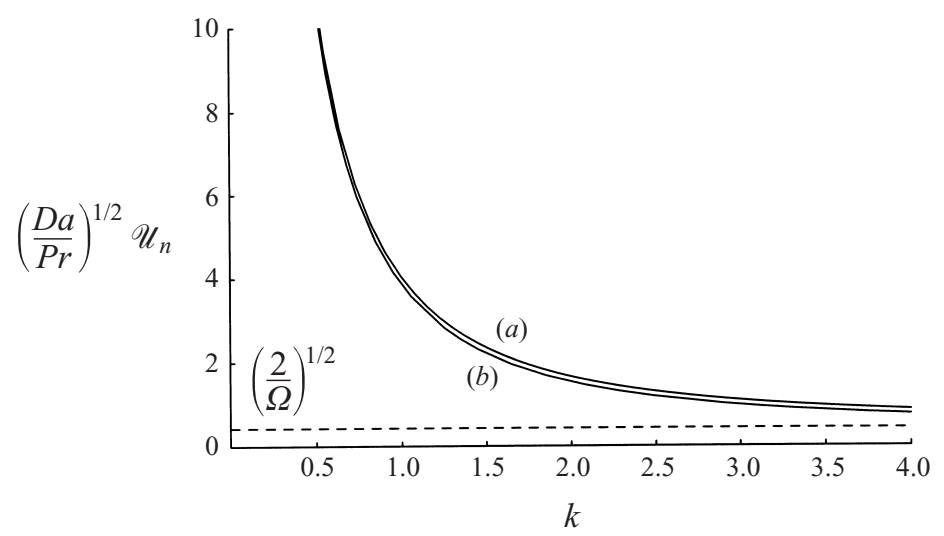

FiguRE 3. The neutral curve ( $a$ ) is obtained numerically from the full Stefan condition ( $3.16 a)$; curve $(b)$ is obtained by ignoring the perturbed heat flux from the melt $(3.18 a)$. The curves are plotted for $D a=10^{-8}, \operatorname{Pr}=10, \Omega=11$ and $\theta_{\infty}=0.1$. Ignoring the perturbed heat flux from the melt gives a good estimate for the neutral curve. Also shown is the asymptote of curve $(b)$ as $k \rightarrow \infty$, this corresponds to the critical flow rate required for instability.

heat flux from the melt then the critical flow rate is

$$
\mathscr{U}_{c r i t}=\left(\frac{2 P r}{D a \Omega}\right)^{1 / 2} \text {. }
$$

This shows that the critical flow rate is inversely proportional to the square root of the permeability.

Consider the neutral curves in figure 3 . We see selectivity at small $k$ (large wavelengths $\lambda$ ) since, as the mush-melt interface flattens, the Bernoulli effect weakens and the stabilizing effect of thermal diffusion dominates. The most significant feature of the neutral curve is the lack of selection at large $k$. This means we could expect growth on infinitesimally small lengthscales, which is not observed nor expected. Our asymptotic assumptions and the averaging procedure used to obtain the mushy-layer equations and Darcy's law will break down at sufficiently small $\lambda$ and we might also expect new physical effects to become important such as pressure-freezing. Inserting new physics into the analysis could generate selection at small wavelengths but we shall see that this is unnecessary.

By abandoning a no-slip (or small-slip) condition at the mush-melt interface, we have allowed relatively large flows within the mushy layer. The destabilizing effect of this flow can dominate over the stabilizing effect of thermal diffusion at all wavenumbers $k>0$. If we consider a viscous melt then there is a viscous boundary layer at the mush-melt interface. The flows induced within the mushy layer are weaker and flows with wavelengths small compared to the depth of the viscous boundary layer are suppressed. The suppression of small-wavelength flows in the mushy layer is because perturbations of the mush-melt interface with wavelengths smaller than the viscous boundary layer depth are relatively unaffected by the large far-field flow in the melt. This is elaborated upon and quantified in $\S 5$.

\section{Further approximations of the mushy layer}

Before proceeding to a more complete analysis of the viscous fluid flow, we simplify the mathematical description of the mushy layer as follows. 
We consider the limit of large Stefan number

$$
\mathscr{S}=\frac{\bar{S}}{\epsilon},
$$

which is of $O(1 / \epsilon)$, and assume that the solid fraction in the mushy layer is a constant $\phi^{m}$. This second assumption, which, though arbitrary, has led to good predictive models of the evolution of mushy layers (e.g. Huppert \& Worster 1985) eliminates the need for the solute conservation equation, any dependence on $\mathscr{C}$ and the solid fraction boundary condition. In particular, this implies that the earlier assumption that $\mathscr{C} \gg 1$ is relaxed. The effect of these two approximations on the Stefan condition is to transform it to

$$
\phi^{m} \mathscr{S} \sigma \zeta_{1}=\left[\zeta_{1} \theta_{0}^{\prime \prime}+\mathrm{D} \theta_{1}\right]_{l}^{m} .
$$

This equation implies that $\sigma=O(1 / \mathscr{S})=O(\epsilon)$, which implies the temperature fields are sufficiently close to the equilibrium profiles determined by the boundary conditions that the time dependences in the field equations should be ignored to $O(\epsilon)$. Because of this, we shall refer to these two approximations as the quasi-stationary approximation.

The form of the steady, basic-state solution is essentially unaffected; the only alterations are that $\Omega \rightarrow 1$ (no coupling of the heat and solute conservation equations) and

$$
\theta_{\infty} \rightarrow \tilde{\theta}_{\infty}=\theta_{\infty}+\bar{S} \phi^{m} .
$$

The new perturbation equation in the mushy layer is

$$
\left(\mathrm{D}^{2}+\mathrm{D}-k^{2}\right) \theta_{1}=w_{1} \mathrm{D} \theta_{0}
$$

and we do not need the equation in the melt since we ignore the perturbed heat flux from this region.

Consider the effect of these approximations on the stability analysis of the preceding section. We ignore the perturbed heat flux in the melt so that we have a direct instability and the neutral curve is determined by the solution to (4.2) for $\mathscr{U}$ where $\sigma \equiv$ 0 . The solution is given by (3.18a) with $\Omega \rightarrow 1$ and $\theta_{\infty} \rightarrow \tilde{\theta}_{\infty}$. These approximations thus preserve the essential aspects of the instability for the case of uniform flow in the melt. We take this as our justification for their adoption in the next section.

\section{Linear stability analysis with shear flow in a viscous melt}

In this section we present the linear stability analysis of the mush-melt interface treating the melt as a viscous fluid governed by the Navier-Stokes equations. In order to make the analysis analytically tractable, we employ the quasi-stationary approximation and ignore the perturbed heat flux from the melt. We expect that the effect of these approximations is to underestimate slightly the flow rate in the melt required to induce instability and to remove the translation of the interface due to the heat advecting into the interface from the melt.

We first describe the steady, basic-state and perturbed flows in the melt and mushy layer. We substitute the solution for the mushy-layer flow into the perturbed temperature equation which is then solved subject to boundary conditions. The growth rate is given by the Stefan condition, which is used to determine the neutral curve.

The Navier-Stokes equations (2.7) admit a steady solution in the moving frame of reference

$$
\boldsymbol{u}_{0}=\left(\mathscr{U}_{\infty}\left(1-\mathrm{e}^{-\left(z-\zeta_{0}\right) / P r}\right), 0\right),
$$


where $\operatorname{Pr}=v / \kappa$ is the Prandtl number, the ratio of kinematic viscosity to thermal diffusivity. This is the asymptotic suction profile used by Forth \& Wheeler (1992) in a related analysis and discussed in Drazin \& Reid (1981). It does not induce any flow in the mushy layer, which remains stagnant in the basic state. The perturbed steady-state Navier-Stokes equations are

$$
-\mathrm{D} \boldsymbol{u}_{1}+\boldsymbol{u}_{0} \cdot \nabla \boldsymbol{u}_{1}+\boldsymbol{u}_{1} \cdot \nabla \boldsymbol{u}_{0}=-\frac{1}{\rho} \nabla p_{1}+\operatorname{Pr} \nabla^{2} \boldsymbol{u}_{1},
$$

where the first term is a pseudo-advective term due to the movement of the reference frame. After some elementary manipulations to eliminate the pressure and using incompressibility, we obtain the equations describing the perturbed flow in the melt

$$
\begin{gathered}
\left\{\operatorname{Pr} \nabla^{4}+\nabla^{2} \mathrm{D}-u_{0} \nabla^{2} \frac{\partial}{\partial x}+u_{0}^{\prime \prime} \frac{\partial}{\partial x}\right\} w_{1}=0, \\
\nabla \cdot \boldsymbol{u}_{1}=0 .
\end{gathered}
$$

Note that $\partial w_{1} / \partial x=\mathrm{i} k w_{1}$, so that $(5.3 a)$ is an ordinary differential equation. The first term in $(5.3 a)$ is the viscous Stokes operator, which dominates within the viscous boundary layer, the second term is due to the movement of the reference frame and the remaining terms are due to advection.

The flows in the melt and mushy layer are linked by continuity of normal mass flux $(2.9 b)$ and continuity of pressure $(2.9 c)$. In order to determine the flow in the melt, we ignore the relatively insignificant vertical transport into the mushy layer. Combining this with the no-slip condition, we have

$$
\boldsymbol{u}=\mathbf{0} \quad(z=\zeta)
$$

in the melt. This implies to $O(\epsilon)$ that

$$
w_{1}=0, \mathrm{D} w_{1}=\mathrm{i} k \zeta_{1} u_{0}^{\prime} \quad\left(z=\zeta_{0}\right),
$$

which are the boundary conditions at the mush-melt interface. An extended discussion of the boundary condition appropriate for flow past a wavy boundary may be found in Tuck \& Kouzoubov (1995). Far from the mush-melt interface the perturbed flow in the melt must be zero,

$$
\lim _{z \rightarrow \infty} w_{1}=0, \lim _{z \rightarrow \infty} \mathrm{D} w_{1}=0 .
$$

The velocity $w_{1}$ is the solution to the fourth-order ordinary differential equation (5.3a) subject to (5.5) and (5.6). The horizontal component of the perturbed velocity is obtained from this solution using $(5.3 b)$.

The pressure exerted at the mush-melt interface is determined from (5.2) to be

$$
p_{1}=\frac{\operatorname{Pr}\left(\mathrm{D}^{2}-k^{2}\right) \mathrm{D} w_{1}+\mathrm{D}^{2} w_{1}}{k^{2}},
$$

where all derivatives are evaluated at $z=\zeta_{0}$. The imaginary part of the pressure causes a flow which leads to translation of the mush-melt interface; the real part of the pressure drives a potentially destabilizing flow. If the real part of the pressure is negative then the flow in the mush is sucked in at crests and blown out at troughs, which is potentially destabilizing.

We now discuss the method used to obtain the solution $w_{1}$. We restrict the range of integration to $[0,1]$ by expressing the problem in terms of the coordinate $s=\mathrm{e}^{-\left(z-\zeta_{0}\right) / P r}$. 
This transforms the differential equation and boundary conditions to

$$
\begin{gathered}
\left\{\left[\left(P^{-3}\right) s^{4}\right] \mathrm{D}^{4}+\left[\left(5 P r^{-3}\right) s^{3}\right] \mathrm{D}^{3}\right. \\
+\left[\left(4 P r^{-3}-2 k^{2} P^{-1}-\mathrm{i} k \operatorname{Pr}^{-2} \mathscr{U}_{\infty}\right) s^{2}+\left(\mathrm{i} k P r^{-2} \mathscr{U}_{\infty}\right) s^{3}\right] \mathrm{D}^{2} \\
+\left[\left(-2 k^{2} P^{-1}-\mathrm{i} k P r^{-2} \mathscr{U}_{\infty}+k^{2} P^{-1}\right) s+\left(\mathrm{i} k P r^{-2} \mathscr{U}_{\infty}\right) s^{2}\right] \mathrm{D} \\
\left.+\left[\left(P r k^{4}+\mathrm{i} k^{3} \mathscr{U}_{\infty}\right)+\left(-\mathrm{i} k^{3} \mathscr{U}_{\infty}-P^{-2} \mathscr{U}_{\infty} \mathrm{i} k\right) s\right]\right\} w_{1}=0, \\
w_{1}=0, \mathrm{D} w_{1}=0 \quad(s=0), \\
w_{1}=0, \mathrm{D} w_{1}=-\mathrm{i} k \zeta_{1} \mathscr{U}_{\infty} \quad(s=1),
\end{gathered}
$$

where $\mathrm{D} \equiv \mathrm{d} / \mathrm{d} s$. We seek a Frobenius series solution of the form

$$
w_{1}=s^{r} \sum_{j=1}^{\infty} A_{j} s^{j} .
$$

The indicial equation has four solutions for the index $r$, which are

$$
r_{1,2}=\mp k P r, r_{3,4}=\frac{1}{2}(1 \mp R),
$$

where

$$
R=\left(1+4 k \operatorname{Pr}\left(\mathrm{i} \mathscr{U}_{\infty}+k P r\right)\right)^{1 / 2} .
$$

A general solution for $w_{1}$ is a linear superposition of four solutions of the form (5.9) corresponding to the four values of the index $r$. The presence of the four different scales of variation $\left(r_{1}\right.$ to $\left.r_{4}\right)$ makes a numerical integration scheme based on the method of shooting awkward. Since $\operatorname{Re}\left[r_{1,3}\right]<0$ (except at $k=0$ ), the solutions containing $s^{r_{1}, r_{3}}$ diverge as $s \rightarrow 0$ and are thus excluded in order to satisfy $(5.8 b, c)$. The solution is

$$
w_{1}=\lambda_{1} s^{r_{2}} \sum_{j=1}^{\infty} a_{j} s^{j}+\lambda_{2} s^{r_{4}} \sum_{j=1}^{\infty} b_{j} s^{j}
$$

where $\lambda_{1}$ and $\lambda_{2}$ are chosen to satisfy $(5.8 d, e)$ and are

$$
\lambda_{1}=\frac{-\mathrm{i} k \zeta_{1} \mathscr{U}_{\infty}}{\sum_{j=1}^{\infty}\left(r_{2}+j\right) a_{j}-\beta \sum_{j=1}^{\infty}\left(r_{4}+j\right) b_{j}}, \lambda_{2}=-\beta \lambda_{1},
$$

where

$$
\beta=\sum_{j=1}^{\infty} a_{j} / \sum_{j=1}^{\infty} b_{j}
$$

The coefficients $a_{j}$ and $b_{j}$ are determined from the recurrence relations

$$
\begin{gathered}
a_{0}=1 ; a_{j+1}=\frac{\mathrm{i}(j+1) \mathscr{U}_{\infty}}{(j+2)\left(2 j+3-\mathrm{i} \mathscr{U}_{\infty}\right)} a_{j}, \\
b_{0}=1 ; b_{j+1}=\frac{\mathrm{i} k \operatorname{Pr} \mathscr{U}_{\infty}\left(\frac{3}{2}+3 j+j^{2}+\mathrm{i} k \operatorname{Pr} \mathscr{U}_{\infty}+R\left(\frac{3}{2}+j\right)\right)}{(2+j)(2+j+R)\left(\frac{13}{2}+5 j+j^{2}+\mathrm{i} k \operatorname{Pr} \mathscr{U}_{\infty}+R\left(\frac{5}{2}+j\right)\right)} b_{j} .
\end{gathered}
$$

At large $j$ these recursion relations imply $a_{j+1} \sim a_{j} / j$ and $b_{j+1} \sim b_{j} / j^{2}$ and thus the series solution for $w_{1}$ (and hence $p_{1}$ ) is very strongly convergent. For large values of 


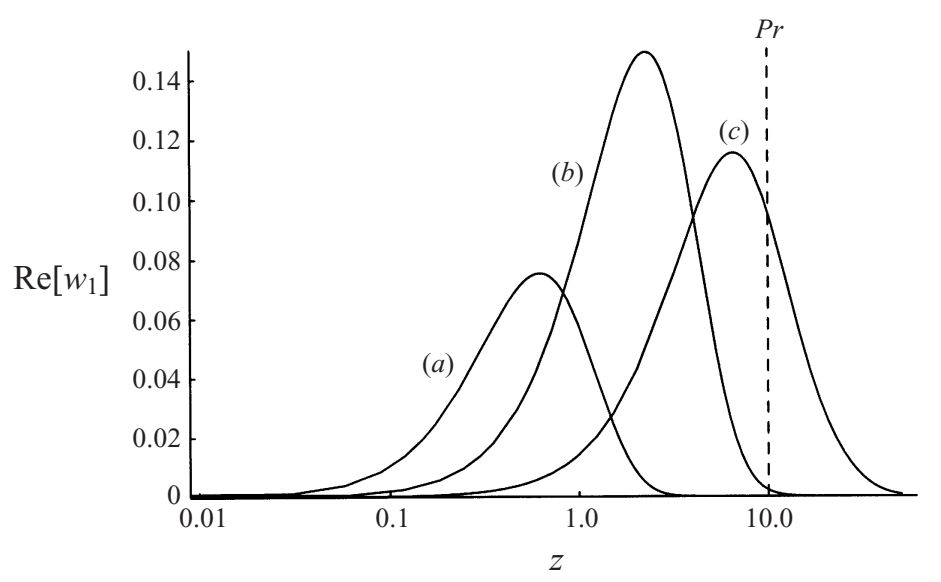

FIGURE 4. The real part of the vertical component of the perturbed velocity for $(a) \lambda=\operatorname{Pr} / 5,(b)$ $\lambda=\operatorname{Pr}$ and $(c) \lambda=5 \operatorname{Pr}$. In $(c)$, the perturbed flow extends well beyond the viscous boundary layer and interacts with the far-field flow.

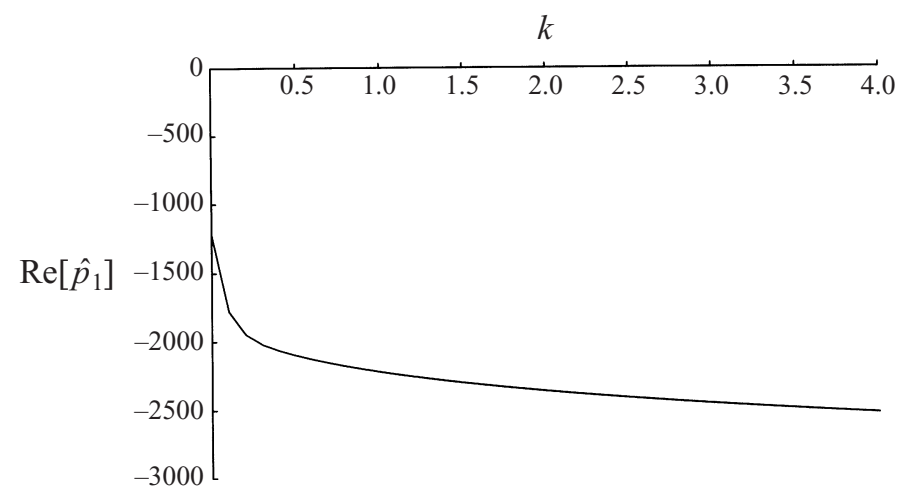

FIGURE 5. The real part of the pressure at the mush-melt interface $\operatorname{Re}\left[\hat{p}_{1}\left(\mathscr{U}_{\infty}, \operatorname{Pr}, k\right)\right]$ versus wavenumber $k$ for $\mathscr{U}_{\infty}=100$ and $\operatorname{Pr}=10$.

the parameters $\mathscr{U}_{\infty}, P r$ and $k$, it is necessary to include many terms in the series and it is important to work with sufficient numerical precision.

The real part of the perturbation flow in the melt drives a potentially destabilizing flow in the mush through the pressure it exerts at the mush-melt interface. This flow, $\operatorname{Re}\left[w_{1}\right]$, is plotted for perturbation wavelengths $\lambda=\operatorname{Pr} / 5, \lambda=\operatorname{Pr}$ and $\lambda=5 / \operatorname{Pr}$ in figure 4 for $\mathscr{U}_{\infty}=10$ and $P r=10$. We see that the flow with perturbation wavelength small compared to the viscous boundary layer depth does not extend beyond the viscous boundary layer whereas the larger-wavelength perturbations extend into the far-field flow.

The pressure is a function of the three parameters $\mathscr{U}_{\infty}, P r$ and $k$ and is obtained from (5.7) using the series solution for $w_{1}$. For convenience, we define $p_{1}=\zeta_{1} \hat{p}_{1}\left(\mathscr{U}_{\infty}, \operatorname{Pr}, k\right)$. The real part of $\hat{p}_{1}$ versus $k$ is plotted in figure 5 for $\mathscr{U}_{\infty}=100$ and $\operatorname{Pr}=10$. As $k \rightarrow 0$ $(\lambda \rightarrow \infty)$, the pressure $\hat{p}_{1} \rightarrow-\mathscr{U}_{\infty}^{2} / \operatorname{Pr}$. Computational restraints prevent investigating the limit $k \rightarrow \infty$, since this would require the evaluation of too many terms in the Frobenius series.

The flow in the mushy layer, described by (3.4), is coupled to the flow in the melt 
by $(2.9 c)$ to give the velocity potential

$$
\Phi_{1}^{m}=-\zeta_{1} \frac{D a}{P r} \hat{p}_{1} \cosh (k z) / \cosh \left(k \zeta_{0}\right) .
$$

This flow is inserted into the quasi-stationary temperature equation (4.4), which is solved subject to the boundary conditions $(3.13 a, b)$ to give the temperature in the mushy region

$$
\begin{aligned}
\theta_{1}^{m}= & \frac{\zeta_{1}(D a / P r) \hat{p}_{1} \theta_{\infty}}{\cosh \left(k \zeta_{0}\right)}\left\{\mathrm{e}^{-\left(z-\zeta_{0}\right)} \cosh (k z)\right. \\
& -\frac{1}{1-\mathrm{e}^{2 Q \zeta_{0}}}\left(2 \mathrm{e}^{(1 / 2+Q) \zeta_{0}} \mathrm{e}^{-\left(z-\zeta_{0}\right) / 2} \sinh \left(Q\left(z-\zeta_{0}\right)\right)\right. \\
& \left.\left.+\cosh \left(k \zeta_{0}\right) \mathrm{e}^{-\left(z-\zeta_{0}\right) / 2}\left(\mathrm{e}^{-Q\left(z-\zeta_{0}\right)}-\mathrm{e}^{Q\left(z+\zeta_{0}\right)}\right)\right)\right\} \\
& +\frac{\zeta_{1} \theta_{\infty}}{1-\mathrm{e}^{2 Q \zeta_{0}}} \mathrm{e}^{-\left(z-\zeta_{0}\right) / 2}\left(\mathrm{e}^{Q\left(z+\zeta_{0}\right)}-\mathrm{e}^{-Q\left(z-\zeta_{0}\right)}\right),
\end{aligned}
$$

where

$$
Q=\frac{1}{2}\left(1+4 k^{2}\right)^{1 / 2} .
$$

The quasi-stationary Stefan condition (4.2) explicitly gives the growth rate $\sigma$. Since $\hat{p}_{1}$ is a complicated function of $\mathscr{U}_{\infty}$, it is convenient to construct a neutral curve of the Darcy number $D a_{n}$ required for instability to ensue. The neutral curve is obtained from (4.2) with $\sigma_{R} \equiv 0$ to be

$$
\begin{aligned}
D a_{n}= & \frac{\operatorname{Pr}}{\operatorname{Re}\left[\hat{p}_{1}\right]}\left(1+\frac{-\frac{1}{2}+Q}{1-\mathrm{e}^{-2 Q \zeta_{0}}}-\frac{\frac{1}{2}+Q}{1-\mathrm{e}^{2 Q \zeta_{0}}}\right) \\
& \times\left\{\frac{-\frac{1}{2}+Q}{1-\mathrm{e}^{-2 Q \zeta_{0}}}\left(-1+\frac{\mathrm{e}^{\left(\frac{1}{2}-Q\right) \zeta_{0}}}{\cosh \left(k \zeta_{0}\right)}\right)-\frac{\frac{1}{2}+Q}{1-\mathrm{e}^{2 Q \zeta_{0}}}\left(-1+\frac{\mathrm{e}^{\left(\frac{1}{2}+\mathrm{Q}\right) \zeta_{0}}}{\cosh \left(k \zeta_{0}\right)}\right)\right. \\
& \left.-1+k \tanh \left(k \zeta_{0}\right)\right\}^{-1},
\end{aligned}
$$

where $\hat{p}_{1}\left(\mathscr{U}_{\infty}, P r, k\right)$ is determined numerically. The viscous boundary layer introduces a phase lag between the mushy-layer flow and the interface, causing the instability to be slightly oscillatory even in the absence of the perturbed heat flux from the melt. In order to treat the oscillatory part of the instability properly, however, we would need to include the perturbed heat flux from the melt, which we have ignored.

The neutral curve is shown in figure 6 for $\mathscr{U}_{\infty}=100, \operatorname{Pr}=10$ and $\tilde{\theta}_{\infty}=1$. The choice for $\tilde{\theta}_{\infty}$ is made so that the depth of the mushy layer is comparable to that considered for an inviscid melt. Also shown, for the equivalent parameter values, is the neutral curve for uniform flow in an inviscid melt expressed in terms of the Darcy number required for instability (dashed line). Two important features arise as a result of including the viscosity of the melt: the instability is weaker (requiring a more porous mush) and there is wavelength selection at large $k$. The instability is strongest at the critical wavenumber $k_{\text {crit }}=2.56$. A sinusoidal circulation cell in the mush occupying the whole of the layer has a wavelength four times the depth of the mushy layer, so that the wavenumber is $2 \pi /\left(4 \zeta_{0}\right) \approx 2.27$. Thus, the critical 


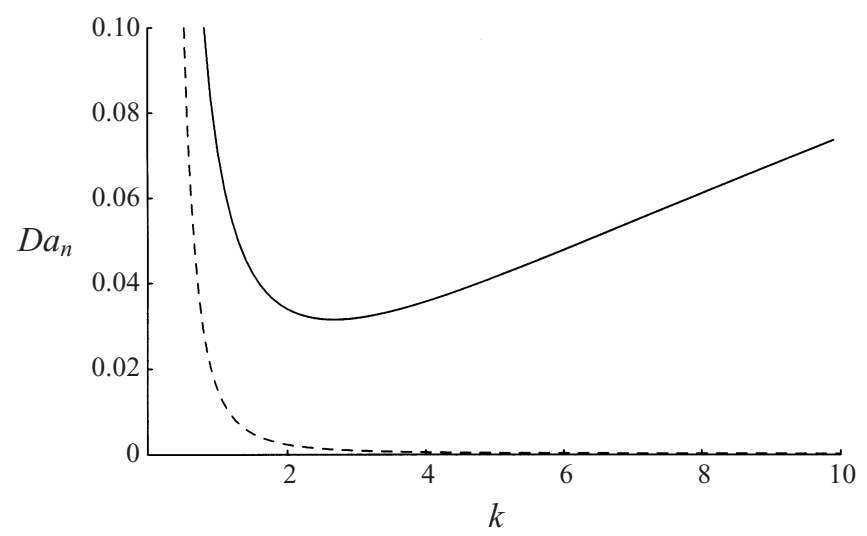

FiguRE 6. Neutral curve for a viscous melt, for $\mathscr{U}_{\infty}=100, \operatorname{Pr}=10$ and $\tilde{\theta}_{\infty}=1$. The crucial feature is the wavelength selection at large $k$. The dashed line shows the inviscid result in terms of a neutral Darcy number for equivalent parameter values.

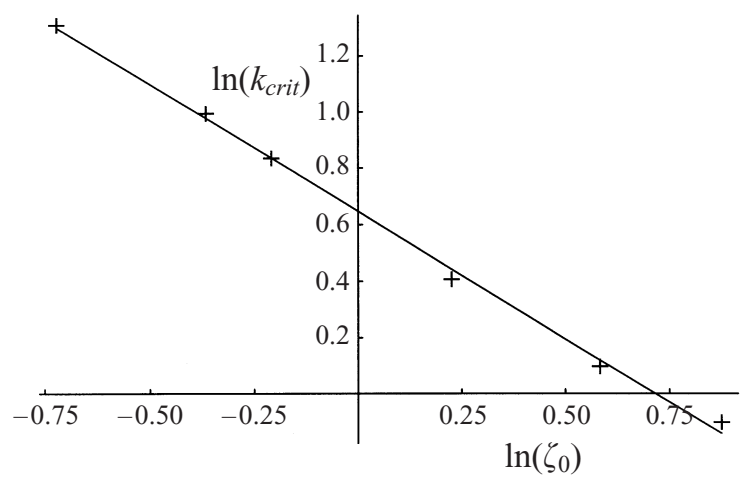

FIGURE 7. $\ln \left(k_{\text {crit }}\right)$ versus $\ln \left(\zeta_{0}\right)$ for $\mathscr{U}_{\infty}=100$ and $\operatorname{Pr}=10$. The curve is very nearly a straight line with a slope of -0.9 .

wavenumber $k_{\text {crit }}$ corresponds to a circulation cell occupying nearly all of the mushy layer. In figure 7, we show a plot of $\ln \left(k_{c r i t}\right)$ versus $\ln \left(\zeta_{0}\right)$ for $\mathscr{U}=100$ and $\operatorname{Pr}=10$. The curve is very nearly a straight line with a slope of $-0.9 \pm 0.02$, indicating that the critical wavenumber is nearly inversely proportional to the mushy-layer depth. This is the result we expect given a critical circulation cell occupying nearly all of the mushy layer. Numerical experiments show $k_{\text {crit }}$ to be independent of the far-field flow in the range $1<\mathscr{U}_{\infty}<10^{3}$ and Prandtl number in the range $0.1<\operatorname{Pr}<20$ for $\tilde{\theta}_{\infty}=1$.

The critical Darcy number required for instability to ensue is $D a_{c r i t}\left(\mathscr{U}_{\infty}, \operatorname{Pr}, \tilde{\theta}_{\infty}\right)$. The variation of $D a_{c r i t} \mathscr{U}_{\infty}^{2}$ with $\mathscr{U}_{\infty}$ for $\operatorname{Pr}=10$ and $\tilde{\theta}_{\infty}=1\left(k_{c r i t}=2.56\right)$ is shown in figure 8 . We see some curious behaviour at low $\mathscr{U}_{\infty}$ (the curve is continuous), which is due to a local maximum of $\left|\operatorname{Re}\left[\hat{p}_{1}\right]\right|$ at about $\mathscr{U}_{\infty}=20$. However, this lies outside the region of validity of the asymptotic analysis used to obtain the kinematic boundary condition at the mush-melt interface (5.4). This is because, for such high Darcy numbers, the vertical transport from the melt into the mushy layer cannot be ignored. We are interested in very small $D a$ and large $\mathscr{U}_{\infty}$. In this regime, we see that $D a_{\text {crit }} \mathscr{U}_{\infty}^{2}$ is only a weak function of $\mathscr{U}_{\infty}$. This implies that the pressure $\hat{p}_{1}$ scales very closely with $\mathscr{U}_{\infty}^{2}$ for large $\mathscr{U}_{\infty}$, which is advection-dominated flow; the advection terms in (5.2) (the second and third terms) largely determine the pressure. 


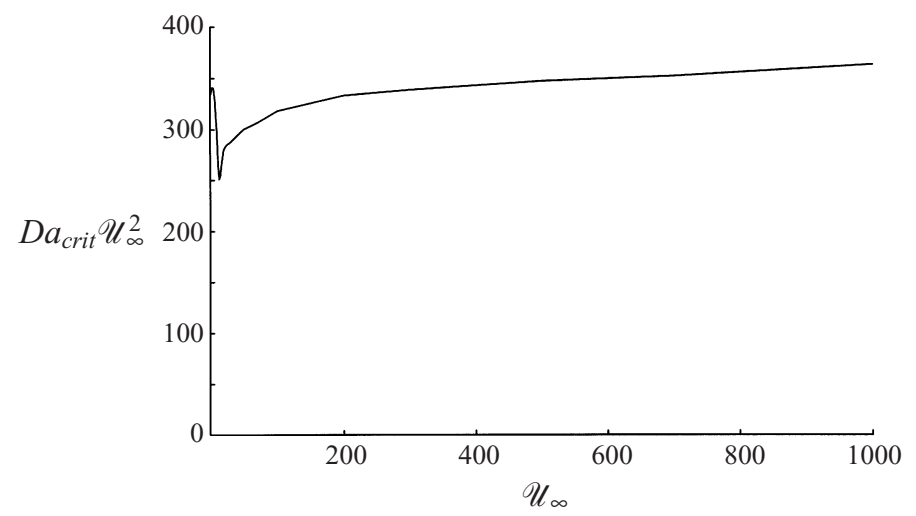

FIGURE 8. The variation of $D a_{\text {crit }} \mathscr{U}_{\infty}^{2}$ with $\mathscr{U}_{\infty}$ for $\tilde{\theta}_{\infty}=1$ and $\operatorname{Pr}=10$.

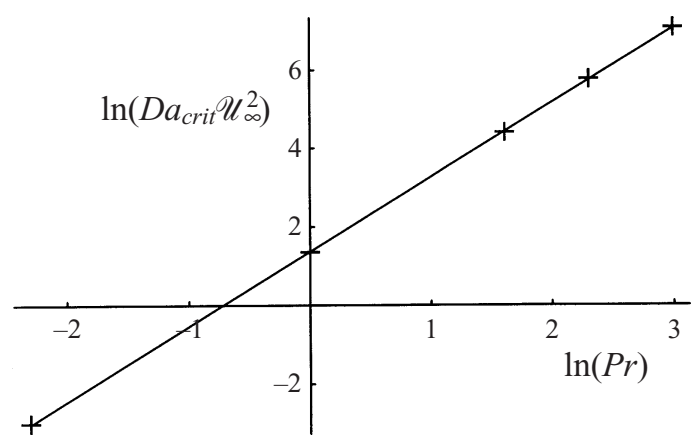

FIGURE $9 . \ln \left(D a_{\text {crit }} \mathscr{U}_{\infty}^{2}\right)$ versus $\ln (P r)$ for $\mathscr{U}_{\infty}=100$ and $\tilde{\theta}_{\infty}=1$. The curve is very nearly a straight line with a slope of 1.9 .

Computational restraints prevent extending the curve out to higher $\mathscr{U}_{\infty}$ but we expect the trends to continue since increasing $\mathscr{U}_{\infty}$ enhances the domination of the advection terms. Note that if we approximate $D a_{c r i t} \mathscr{U}_{\infty}^{2} \sim$ constant at large $\mathscr{U}_{\infty}$ then this implies $\mathscr{U}_{c r i t} \propto 1 / D a^{1 / 2}$, which is the result obtained in the inviscid case.

In figure 9, we plot $\ln \left(D a_{\text {crit }} \mathscr{U}_{\infty}^{2}\right)$ versus $\ln (P r)$ for $\mathscr{U}_{\infty}=100$ and $\tilde{\theta}_{\infty}=1$. The curve is very nearly a straight line with a slope of $1.9 \pm 0.006 \approx 2$, implying that $D a_{\text {crit }}$ is almost proportional to $\mathrm{Pr}^{2}$. This means that $\operatorname{Re}\left[\hat{p}_{1}\right]$ is approximately proportional to $1 / \operatorname{Pr}$, which is also the dependence of $\hat{p}_{1}$ on $\operatorname{Pr}$ obtained exactly in the limit $k \rightarrow 0$.

In order to investigate which feature of the flow in the melt is most important for the instability of the mush-melt interface, we consider basic states of the form

$$
\boldsymbol{u}_{0}=\left(\mathscr{U}_{\infty}\left(1-\mathrm{e}^{-\alpha\left(z-\zeta_{0}\right)}\right), 0\right) \text {. }
$$

If $\alpha=1 / P r$ then our basic state satisfies the steady-state Navier-Stokes equations; this corresponds to the case already investigated. If $\alpha \neq 1 / P r$ then the basic state is time-dependent and the linear stability analysis is quasi-static: we consider the basic state to be frozen in time. Assuming a basic-state flow of this form introduces another parameter into the series solution for the pressure but the analysis differs only slightly from the steady-state case. In figure 10 , we show a plot of $\ln \left(D a_{\text {crit }} \mathscr{U}_{\infty}^{2}\right)$ against $\ln (\alpha)$ for $\mathscr{U}_{\infty}=100, \operatorname{Pr}=10$ and $\tilde{\theta}_{\infty}=1$. The critical wavenumber $k_{\text {crit }}=2.56$ throughout. The curve is very nearly a straight line with a slope of $-0.9 \pm 0.07$. If we consider $D a_{\text {crit }} \mathscr{U}_{\infty}^{2} \sim$ constant at large $\mathscr{U}_{\infty}$ then this implies approximately that 


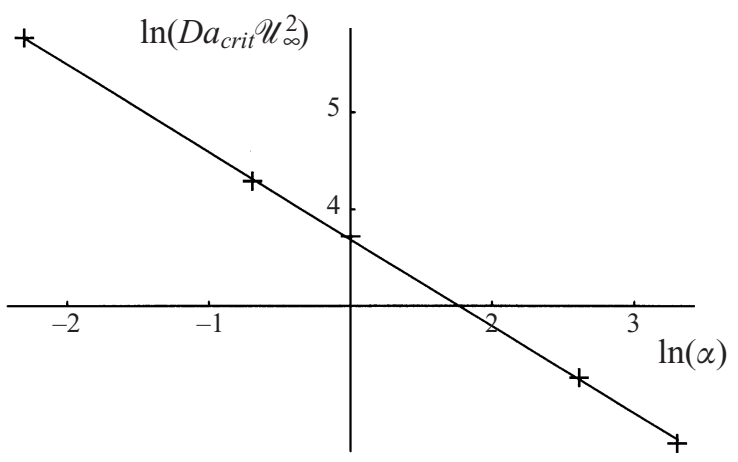

FIGURE $10 . \ln \left(D a_{c r i t} \mathscr{U}_{\infty}^{2}\right)$ versus $\ln (\alpha)$ for $\mathscr{U}_{\infty}=100, \operatorname{Pr}=10$ and $\tilde{\theta}_{\infty}=1$. The curve is very nearly a straight line with a slope of -0.9 .

$\mathscr{U}_{c r i t} \propto \alpha^{-0.45}$. This means $\mathscr{U}_{c r i t} \alpha^{0.45}$ is almost independent of the basic state (through $\alpha)$ and can be considered to be the driving force for the instability. The critical shear at the mush-melt interface is $S_{\text {crit }}=\alpha \mathscr{U}_{\text {crit }}$. Our result suggests that the geometric mean of $S_{\text {crit }}$ and $\mathscr{U}_{\text {crit }}$, which is $\alpha^{1 / 2} \mathscr{U}_{c r i t} \sim \alpha^{0.45} \mathscr{U}_{c r i t}$, is crucial to the instability.

\section{Discussion}

Sea ice forms in polar oceans due to the freezing of seawater and is an extremely important part of the Earth's climate system due to its extent and the role it plays as a barrier to the transfer of heat, mass and momentum between the atmosphere and ocean. It is also important in deep water formation, which drives large-scale ocean circulations. We model sea ice as a mushy layer consisting of almost pure ice immersed in brine (concentrated salt solution). It has been suggested (Wettlaufer 1991) that the relative flow of the upper ocean past sea ice is responsible for a wavy sea-ice-ocean interface due to the instability mechanism of Gilpin et al.(1980). The mechanism presented in this paper might also be responsible for the development of a wavy sea-ice-ocean interface. In this section, we present a short summary of the instability mechanism of Gilpin et al. and compare the potency of this mechanism to that presented in this paper for the case of newly forming sea ice.

Typical sea-ice parameter values for newly forming sea ice are $\Pi=10^{-10} \mathrm{~m}^{2}$, $\mu=1.1 \times 10^{-3} \mathrm{~kg} \mathrm{~m}^{-1} \mathrm{~s}^{-1}$ and $\rho=1.027 \times 10^{3} \mathrm{~kg} \mathrm{~m}^{-3}$ (giving $v=1.07 \times 10^{-6} \mathrm{~m}^{2} \mathrm{~s}^{-1}$ ), $\kappa=1.33 \times 10^{-7} \mathrm{~m}^{2} \mathrm{~s}^{-1}, V=10^{-6} \mathrm{~m} \mathrm{~s}^{-1}$ and $\mathscr{S}=4.2$. These values approximately give $D a=10^{-8}$ and $P r=10$, we choose $\tilde{\theta}_{\infty}=1$ to give an appropriate sea-ice depth of $(\kappa / V) \ln \left(1+1 / \tilde{\theta}_{\infty}\right) \approx 7 \mathrm{~cm}$. The arguments and sources for these parameter values can be found in Feltham (1997). A typical far-field flow rate is $0.1 \mathrm{~m} \mathrm{~s}^{-1}$ (inferred from data in Omstedt \& Wettlaufer 1992), which gives $\mathscr{U}_{\infty} \approx 10^{5}$. Computational restraints prevent calculation of the neutral curves much beyond $\mathscr{U}_{\infty} \approx 10^{3}$. However, we can extend the curve in figure 8 out to $\mathscr{U}_{\infty} \approx 10^{5}$ (extrapolating from a log-log plot) on the basis that we are dealing with advection-dominated flow. By treating $D a_{\text {crit }} \mathscr{U}_{\infty}^{2} \approx 400$ we have, for $D a=10^{-8}$, a dimensional critical far-field flow rate of $0.2 \mathrm{~m} \mathrm{~s}^{-1}$, which is equivalent to a dimensional critical shear of $0.2 \mathrm{~s}^{-1}$. This flow rate is rather high but within the observed range, suggesting that our instability is important. We expect the wavelength of corrugations of the sea-ice-ocean interface to be of the order of the sea-ice depth, with a wavenumber of $25.6 \mathrm{~m}^{-1}$ (corresponding to a wavelength of $0.25 \mathrm{~m}$ ). 


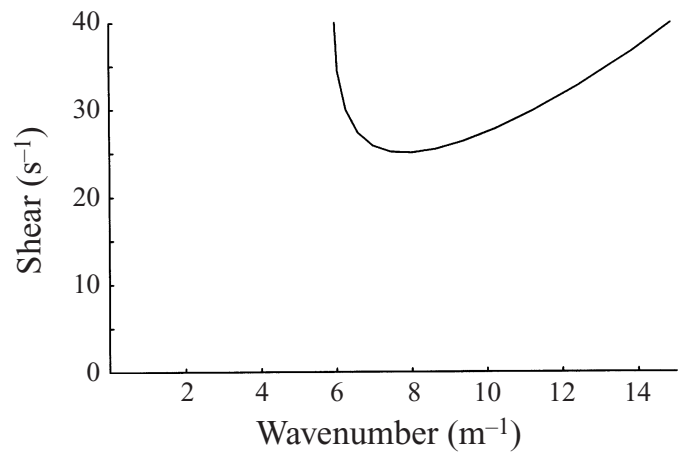

FIGURE 11. Neutral curves showing the dimensional shear at the sea-ice-ocean interface versus dimensional wavenumber for Gilpin et al.'s mechanism. The shear rate required for instability is approximately 125 times larger than for our mechanism and the critical wavenumber is approximately 3 times smaller.

Gilpin et al. present an instability of a plane solid-melt interface due to a turbulent flow in the melt. If the turbulent heat flux into the solid-melt interface is out of phase with incipient corrugations by greater than $\pi / 2$ and less than $\pi$ then the heat flux is enhanced near troughs and retarded near crests. This causes the perturbations to grow and move downstream. In the analysis of Gilpin et al., the heat flux into a wavy icewater interface was determined by fitting experimental data for finite perturbations to an expression determined by Ashton (1972). This expression is used in a linear stability analysis for perturbations to the ice-water interface to determine a neutral curve. In this analysis, the length- and timescales are $v / u^{*}$ and $v / u^{* 2}$ respectively, where $u^{*}$ is the (dimensional) friction velocity, given by

$$
u^{*}=\left(\left.v \frac{\partial U}{\partial z}\right|_{\text {interface }}\right)^{1 / 2},
$$

where $U$ is the local mean flow in the melt. This expression is true for both turbulent and laminar flows though the friction velocity is typically much larger for turbulent flow.

Gilpin et al.'s neutral curves (their figure 7) show the critical Reynolds number $R e=u^{*} d_{0} / v$ (based on the friction velocity and constant ice depth $d_{0}$ ) against the scaled wavenumber $k^{+}=k v / u^{*}$ ( $k$ is the dimensional wavenumber) for several situations. We are interested in their $G=1$ curve which corresponds to a steady, stationary, basic state with a constant ice depth. The choice of scalings implies that the critical wavelength of the instability scales on the depth of the ice layer; since the cause of the instability is the turbulent heat flux from the melt, this seems inappropriate. Since Gilpin et al. used an expression for turbulent heat flux into an interface with finite perturbations, their analysis is inconsistent. In practice, they were only able to trigger their instability by melting large (several centimetre) perturbations into an ice-water interface.

For a particular set of parameter values, we can convert Gilpin et al.'s neutral curve into a plot of shear $S=\partial U / \partial z$ at the interface against a dimensional wavenumber $k$ and compare this with the critical shear for our mechanism $\left(0.2 \mathrm{~s}^{-1}\right)$. We set $d_{0}=7 \mathrm{~cm}$ and $v=1.07 \times 10^{-6} \mathrm{~m}^{2} \mathrm{~s}^{-1}$ and obtain the shear using (6.1). In figure 11, we plot the neutral curve of shear versus wavenumber for Gilpin et al.'s mechanism, which 
we see requires a minimum shear of $25 \mathrm{~s}^{-1}$ at the critical wavenumber of $8 \mathrm{~m}^{-1}$ (corresponding to a wavelength of $0.79 \mathrm{~m}$ ). For a laminar velocity profile such as we have assumed in our analysis, this corresponds to a far-field flow rate of $25 \mathrm{~m} \mathrm{~s}^{-1}$, very much higher than observed values.

Since our instability mechanism requires a shear about 125 times smaller than Gilpin et al.'s for newly forming sea ice, we conclude that our instability mechanism is likely to be more important. Also, since each mechanism predicts a different wavelength of corrugation, this may be a way to distinguish which mechanism is responsible for any observed corrugations.

\section{Conclusions}

The most important result is that a laminar flow in the melt can trigger an instability of a mush-melt interface by inducing a flow in the porous mush and melt which deforms the isotherms. It was shown that the perturbed heat flux from the melt into the mush-melt interface had almost the sole effect of translating corrugations of the interface and thus was neutral to the instability. The perturbed heat flux in the mushy layer can cause perturbations to grow and drives the instability. The coupling of the thermal and solutal fields in the mushy layer is inessential for the instability and this coupling was removed in the consideration of a viscous melt. The strength of the instability varies with the perturbation wavelength due to the weakness of the Bernoulli effect at large $\lambda$ (the interface flattens) and the presence of a viscous boundary layer, which causes a small- $\lambda$ cut-off.

For a viscous melt, $D a_{c r i t} \mathscr{U}_{\infty}^{2}$ was found to be a weak function of $\mathscr{U}_{\infty}$ for large $\mathscr{U}_{\infty}$, which corresponds to advection-dominated flow in the melt. This implies that $\mathscr{U}_{c r i t}$ is almost proportional to $1 / D a^{1 / 2}$, which is the exact result obtained for inviscid flow. For a viscous melt, $D a_{\text {crit }}$ is approximately proportional to $\mathrm{Pr}^{2}$. The critical wavenumber approximately scales on the reciprocal of the mushy-layer depth and is independent of the flow parameters.

By considering a set of basic states with a flow in the melt parallel to the mushmelt interface of the form $\mathscr{U}_{\infty}\left(1-\mathrm{e}^{-\alpha\left(z-\zeta_{0}\right)}\right)$, we gained insight into the features of the basic-state flow essential for the instability. It seems both the far-field flow in the melt and the shear at the mush-melt interface play a role but that their geometric mean may be the crucial controlling parameter.

Throughout this study we have ignored the existence of the hydrodynamic modes which can develop. For flow past a plane interface, numerical solutions of the OrrSommerfeld equation predict that these modes are triggered at Reynold numbers greater than 47,047 (Drazin \& Reid 1981) although the waviness of the interface may lower this threshold. An interesting study would be to investigate the interactions between the hydrodynamic modes and the morphological modes presented here.

For newly-forming sea ice, our instability mechanism requires a shear about 125 times smaller than Gilpin et al.'s mechanism, which suggests it is more important. Our instability mechanism is likely to result in corrugations of the sea-ice-ocean interface with a wavelength comparable to the sea-ice depth.

We are grateful for discussions with J. S. Wettlaufer. M.G.W. is supported by the National Environment Research Council. 
Anderson, D. M. \& Worster, M. G. 1996 A new oscillatory instability in a mushy layer during the solidification of binary alloys. J. Fluid Mech. 307, 245-267.

Ashton, G. D. 1972 Turbulent heat transfer to wavy boundaries. Proc. 1972 Heat Transfer Fluid Mech. Inst., pp. 200-213.

Ashton, G. D. \& Kennedy, J. F. 1972 Ripples on underside of river ice covers. Proc. ASCE 98 (HY9), 1603-1624.

Beavers, G. S. \& JosePh, D. D. 1967 Boundary conditions at a naturally permeable wall. J. Fluid Mech. 30, 197-207.

Drazin, P. G. \& ReID, W. H. 1981 Hydrodynamic Stability. Cambridge University Press.

Emms, P. W. \& Fowler, A. C. 1994 Compositional convection in the solidification of binary alloys. J. Fluid Mech. 262, 111-139.

Fang, Q. T., Glicksman, M. E., Coriell, S. R., McFadden, G. B. \& Boisvert, R. F. 1985 Convective influence on the stability of a cylindrical solid-liquid interface. J. Fluid Mech. 151, 121-140.

Feltham, D. L. 1997 Fluid dynamics and thermodynamics of sea ice. PhD thesis, University of Cambridge, UK.

Forth, S. A. \& Wheeler, A. A. 1992 Coupled convective and morphological instability in a simple model of the solidification of a binary alloy, including shear flow. J. Fluid Mech. 236, 61-94.

Fowler, A. C. 1985 The formation of freckles in binary alloys. IMA J. Appl. Maths 35, 159-174.

Gilpin, R. R., Hirata, T. \& Cheng, K. C. 1980 Wave formation and heat transfer at an ice-water interface in the presence of a turbulent flow. J. Fluid Mech. 99, 619-640.

Hills, R. N., Loper, D. E. \& Roberts, P. H. 1983 A thermodynamically consistent model of a mushy zone. Q. J. Mech. Appl. Maths 36, 505-539.

Huppert, H. E. 1990 The fluid mechanics of solidification. J. Fluid Mech. 212, 209-240.

Huppert, H. E. \& Worster, M. G. 1985 Dynamic solidification of a binary melt. Nature 314, 703-707.

Omstedt, A. \& Wettlaufer, J. S. 1992 Ice growth and oceanic heat flux: models and measurements. J. Geophys. Res. 97 (C6), 9383-9390.

ÖzIsıK, M. N. \& Mulligan, J. C. 1969 Transient freezing of liquids in forced flow inside circular tubes. J. Heat Transfer 91, 385-389.

Tuck, E. O. \& Kouzoubov, A. 1995 A laminar roughness boundary condition. J. Fluid Mech. 300, $59-70$.

Wettlaufer, J. S. 1991 Heat flux at the ice-ocean interface. J. Geophys. Res. 96, 7215-7236.

Wettlaufer, J. S., Worster, M. G. \& Huppert, H. E. 1997 The phase evolution of young sea ice. Geophys. Res. Lett. 24, 1251-1254.

Worster, M. G. 1986 Solidification of an alloy from a cooled boundary. J. Fluid Mech. 167, 481-501.

Worster, M. G. 1991 Natural convection in a mushy layer. J. Fluid Mech. 224, 335-359.

Worster, M. G. $1992 a$ Instabilities of the liquid and mushy regions during solidification of alloys. J. Fluid Mech. 237, 649-669.

Worster, M. G. 1992b The Dynamics of Mushy Layers. NATO Advanced Study Institute Series E, Applied Sciences, vol. 219, pp. 113-138. Kluwer. 